\title{
Análise do comportamento de acadêmicos de Odontologia em tempos de pandemia do novo coronavírus a partir das imagens do Instagram
}

\section{Analysis of the behavior of dental students in times of pandemic of the new coronavirus from Instagram images}

\author{
Francisca Mariane Martins Monte ${ }^{1}$, Mauro Vinicius Dutra Girão ${ }^{2}$ \\ ${ }^{1}$ Centro Universitário Inta (UNINTA), Sobral, Ceará, Brasil, Brasil. ORCID: https://orcid.org/0000-0003-3749-0923 \\ ${ }^{2}$ Centro Universitário Inta (UNINTA), Sobral, Ceará, Brasil, Brasil. ORCID: http://orcid.org/0000-0001-6577-3168
}

Autor para correspondência/Mail to: Francisca Mariane Martins Monte, fmarianemm@gmail.com

Recebido/Submitted: 26 de março de 2021; Aceito/Approved: 13 de julho de 2021

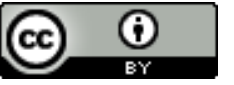

Copyright (c) 2022 Monte \& Girão. Todo o conteúdo da Revista (incluindo-se instruções, política editorial e modelos) está sob uma licença Creative Commons Atribuição 4.0 Internacional. Ao serem publicados por esta Revista, os artigos são de livre uso em ambientes educacionais, de pesquisa e não comerciais, com atribuição de autoria obrigatória. Mais informações em http://revistas.ufpr.br/atoz/about/submissions\#copyrightNotice.

\begin{abstract}
Resumo
Introdução: O mundo encontra-se em uma crise sanitária causada pelo SARS-CoV2, que fez com que governantes decretassem medidas para enfrentamento e contenção da infecção humana, que mudaram os hábitos da população, estes podem ser acompanhados por meio de postagens em Redes Socias on-line. Objetivo: Analisar o comportamento de acadêmicos da área da saúde em relação ao cumprimento dos decretos estaduais para evitar a disseminação da COVID-19 e comportamentos de risco por meio de imagens publicadas na Rede Social Instagram. Metodologia: Pesquisa exploratória, quantitativa-descritiva com abordagem infodemiológica que analisa o comportamento de estudantes de Odontologia em relação ao cumprimento dos decretos do governo do Estado do Ceará para a contenção da infecção por coronavírus provocada pelo SARS-CoV2 por meio de fotos postadas na Rede Social Instagram. A mineração e análise das imagens ocorreram durante um período de cinco dias ininterruptos, realizadas no período que ocorreria o carnaval de 2021, compreendido do dia 13 ao dia 17 de fevereiro. Resultados: A amostra foi composta por 47 perfis de acadêmicos de Odontologia. Desses, 12 apresentaram algum comportamento de risco, em sua maioria adotado por mulheres, sendo o mais frequente o não cumprimento do distanciamento social. Os comportamentos de risco foram identificados em $80 \%$ dos dias analisados. Conclusão: Foram observados comportamentos de risco por acadêmicos da área da saúde. Mesmo sendo a minoria da amostra estudada, os acadêmicos identificados adotando comportamento de risco podem ter sido potenciais disseminadores do SARS-CoV-2, não podendo ser confirmado por meio de imagens.
\end{abstract}

Palavras-chave: Estudantes de Odontologia; Infecções por Coronavírus; Redes Sociais.

\begin{abstract}
Introduction: The world is in a health crisis caused by SARS-CoV2, which caused governments to enact measures to cope with and contain the human infection that changed habits of the population; these can be followed through posts on Social Networks online. Objective: To analyze the behavior of health students about compliance with state decrees to prevent the dissemination of COVID-19 and risk behaviors through images published on the Social Network Instagram. Methodology: Exploratory, quantitative-descriptive research with an infodemiological approach that analyzes the behavior of dentistry students about compliance with the decrees of the government of the State of Ceará for the containment of coronavirus infection caused by SARS-CoV2 through photos posted on the Social Network Instagram. The mining and analysis of the images occurred during five uninterrupted days, held in the period that would take place at the carnival of 2021, between the 13th and the 17th of February. Results: The sample consisted of 47 profiles of dentistry students. Of these, 12 presented some risk behavior, mostly adopted by women, the most frequent being non-compliance with social distancing. It identifies risk behaviors on $80 \%$ of the days analyzed. Conclusion: It observes risk behaviors by health students. Even though the minority of the sample studied, the students identified adopting risky behavior may have been potential disseminators of SARS-CoV-2, and cannot be confirmed by imaging.
\end{abstract}

Keywords: Students, Dental; Coronavirus infection; Social network.

\section{INTRODUÇÃO}

Em dezembro de 2019 foi identificado na China o novo coronavírus (SARS-CoV2) causador da COVID-19, doença respiratória de rápida disseminação e letalidade. Desde a sua descoberta passou a se disseminar pelo mundo caracterizando uma pandemia que continua vitimando em larga escala (Organização Mundial de Saúde, 2021).

O primeiro caso de COVID-19 no Brasil foi relatado em fevereiro de 2020 e, desde então, o país se encontra em uma crise sanitária que vem alterando o cotidiano dos brasileiros, gerando colapsos em sistemas de saúde e, consequentemente, a morte de milhares de pessoas (França, Oliveira Araújo, e Silva, 2020; P. V. D. Silva, 2020; Lehmkuhl e da Silva, 2020; F. C. C. Silva, 2020; Inomata, dos Passos, Pintro, Sena, e Bedin, 2020).

O número de casos confirmados de COVID-19 em no Brasil até junho de 2021 ultrapassava 17 mil e o número de mortes ultrapassava 480 mil; destes, mais de 840 mil casos confirmados e mais de 21 mil óbitos ocorreram no Estado do Ceará. (Organização Mundial de Saúde, 2021; Secretaria de Saúde do Estado do Ceará, 2021). Lehmkuhl e da Silva (2020) ainda sugerem um possível subdiagnóstico e subregistros de óbitos por COVID-19 no Brasil. 
Seguindo as recomendações da OMS, o Governador do Estado do Ceará decretou situação de emergência em saúde no âmbito estadual desde março de 2020, dispondo sobre uma série de medidas para enfrentamento e contenção da infecção humana provocada pelo novo coronavírus com o objetivo de preservar a saúde da população. Entretanto, houve um crescente aumento do número de casos de pessoas infectadas (Secretaria de Saúde do Estado do Ceará, 2021).

Diante da gravidade da situação, o Governo do Estado do Ceará vem prorrogando as medidas e, em janeiro de 2021, a fim de reduzir o aumento do número de casos no período de Carnaval, decretou a proibição de quaisquer festas ou eventos de pré-carnaval e carnaval, em ambientes abertos ou fechados, promovidos por iniciativa pública ou particular, bem como a da extinção do ponto facultativo durante esse período a fim de coibir aglomerações, bem como quanto à obrigatoriedade do uso de máscaras (Decreto n. 33.927, 2021).

Para acompanhar o cumprimento das medidas relativas ao âmbito da saúde, faz-se necessário realizar um acompanhamento por meio da vigilância epidemiológica. Uma estratégia para a vigilância epidemiológica é a Infodemiologia, a ciência da distribuição e de determinantes de informação em meio eletrônico, especificamente a Internet, que sejam de interesse da saúde pública e políticas públicas, que permite analisar o comportamento da comunicação e publicação na Internet, e se utiliza de sites, grupos de discussão e mídias sociais para desenvolver, coletar e avaliar potenciais indicadores epidemiológicos. Desde que as informações estejam disponíveis em formato eletrônico, elas podem ser coletadas e analisadas automaticamente por métodos estatísticos e assim representar o comportamento dos usuários e eventos relevantes de saúde pública (Bragazzi et al., 2016; Eysenbach, 2009; Mavragani, Ochoa, e Tsagarakis, 2018).

A análise de como as pessoas acessam, pesquisam, comunicam e compartilham informações na Internet pode fornecer informações valiosas para comportamento relacionado à saúde das populações, que antes não eram disponíveis. Sabendo que o comportamento em relação à saúde pode ser estudado por meio de informações on-line, o monitoramento torna-se um método de pesquisa que cresce significativamente (Eysenbach, 2009; Mavragani et al., 2018; Tana, 2019).

Os avanços tecnológicos têm criado meios e suportes para produção, compartilhamento e acesso à informação. Dentre esses meios, destacam-se as plataformas de Redes Sociais on-line, as quais são de fácil acesso, de simples manuseio e de grande alcance das informações disponibilizadas. Permitindo acesso dinâmico a informações mais diversas do comportamento humano, dos interesses individuais e coletivos, e permitem acompanhar atitudes prejudiciais à saúde e identificar o comportamento de usuários em relação à pandemia de coronavírus (Araujo e Oliveira, 2020; Carvalho e Dallagassa, 2014; A. P. Santos e Nascimento, 2021; P. V. D. Silva, 2020; L. R. L. Silva e Botelho-Francisco, 2018; I. O. Silva e Gouveia, 2021).

Dentre as plataformas de Redes Sociais, existe o Instagram, criado em 2010, que tem a publicação e o compartilhamento de imagens como a principal finalidade de sua utilização. A postagem de fotos instantâneas através desta plataforma de Rede Social, intensifica-se ao passar dos anos, tendo 1 bilhão de usuários postando diariamente em torno de 95 milhões de fotos. O Brasil é o segundo país com mais usuários, com perspectivas de aumento (Grimaldi, Rosa, Loureiro, e Oliveira, 2019; Pires, 2013; Rezer, Knoll, e Ghisleni, 2018; Rodrigues, 2018).

Uma compreensão avançada dos comportamentos nas plataformas de Redes Sociais pode ajudar a traçar estratégias de gestão em saúde, aumentar a vigilância epidemiológica tradicional e ajudar a alcançar a meta de prevenção e controle de doenças que afetam uma grande parcela da população (Tana, 2019; Xu et al., 2019).

As necessidades da população de serviços de saúde continuam mesmo em tempo de pandemia. Os profissionais vinculados a esse serviço estão mais sujeitos ao contágio com organismos virulentos e patogênicos. Dentre eles, um dos que apresentam alto risco de contaminação por COVID-19 são os Cirurgiões Dentistas (CD) por trabalharem em contato com fluídos orais. O SARS-Cov-2 é abundante nas secreções salivares e nasofaríngeas de afetados, que favorecem sua disseminação por transmissão direta, sendo necessária a adoção de uma rotina rigorosa de biossegurança a fim de reduzir os riscos de infecções (Maciel, da Silva Sobrinho, e Medrado, 2020; K. F. Santos e Barbosa, 2020).

Poucos estudos abordam a contribuição do acadêmico de saúde como potencial transmissor de COVID-19, desta forma o presente estudo objetivou identificar por meio da análise de imagens publicadas na rede social Instagram o comportamento de acadêmicos de Odontologia em relação ao cumprimento dos decretos do Governo do Estado do Ceará determinando o isolamento social e outras medidas preventivas direcionadas para evitar a disseminação da COVID-19.

\section{MÉTODO}

O presente artigo tem como problema a seguinte questão: Como acadêmicos da área da saúde, do curso de Odontologia do Centro Universitário UNINTA, lidam com os decretos relativos às medidas preventivas da disseminação da COVID-19 durante o período de carnaval? 
Assim, foi realizado um estudo exploratório quantitativo-descritivo de mineração de dados de domínio público disponíveis na rede social Instagram, caracterizando um estudo Infodemiológico transversal baseado na demanda em tempo real que, segundo de Eysenbach (2009), é realizado por meio de coleta de dados provenientes de mecanismos de busca em ferramentas da internet que caracterizam o comportamento coletivo por busca de informações.

Um método passivo, analisando tendências na Internet de forma automática sem envolver ativamente os usuários. Como também, um método bruto que é realizado usando um termo de pesquisa em um mecanismo de busca, que fornece um número absoluto de ocorrências ao longo do tempo.

A mineração de dados é uma ferramenta que fornece dados de forma rápida e com baixo custo. No presente estudo, mineração de dados foi realizada seguindo as etapas propostas por Carvalho e Dallagassa (2014), sendo elas a coleta de dados disponíveis que representem de forma confiável o mundo real, consistência e armazenamento, análise, identificação de similaridades ou associações para posterior extração de informações por profissionais capacitados nas respectivas técnicas.

Optou-se por restringir o estudo à plataforma de Rede Social Instagram por tornar o trabalho mais exequível, pelo fato desta Rede Social ser uma das mais utilizadas e ter a postagem de fotos como uma de suas principais características e pelo conteúdo das postagens representarem o comportamento social do indivíduo consigo mesmo e a sua comunidade (ConnectSafely, 2021).

O material de análise do presente artigo consistiu de fotos do cotidiano social durante o feriado de carnaval de 2021 postadas em perfis da rede social Instagram de acadêmicos de Odontologia do Centro Universitário UNINTA.

O UNINTA é uma Instituição de Ensino Superior localizada no noroeste do Estado do Ceará, a cerca de $220 \mathrm{~km}$ da capital. A escolha por essa instituição de ensino se deu por conveniência, como também pelo alarmante número de casos e óbitos por COVID19 no Estado.

O Curso de Odontologia do UNINTA foi autorizado pelo Ministério da Educação com nota máxima, sendo o primeiro curso de Odontologia privado da Região Noroeste do Estado do Ceará. Possui infraestrutura de laboratórios de ciências básicas, laboratório de habilidades odontológicas, laboratório pré-clínico e clínica odontológica universitária, destacando-se como uma das maiores e mais modernas do Norte-Nordeste do Brasil, atendendo a comunidade por meio de projetos de extensão, monitorias, ligas acadêmicas e campos de estágios municipais e estaduais.

Inicialmente foi consultado o site institucional do Centro Universitário, buscou-se a matriz curricular para identificar quais turmas realizavam atendimento odontológico na clínica universitária. Foram identificadas nove turmas. A partir disso, buscou-se a página oficial do Centro Universitário no Instagram e nela buscou-se os perfis das turmas, sendo identificadas sete. Acessando esses perfis, foram identificados um total de 223 acadêmicos seguidores, desses 47 (21\%) eram perfis públicos.

A identificação dos perfis das turmas e dos acadêmicos seguidores foi realizada por um mesmo pesquisador que verificava se cada perfil atendia aos critérios de inclusão, isto é, se era público, se pertencia a um acadêmico de Odontologia do respectivo Centro Universitário. Por se tratar de um estudo infodemiológico, baseado na demanda, passivo e bruto, os autores limitaram-se aos dados da plataforma de Rede Social Instagram, não tendo acesso aos dados institucionais.

A mineração e análise das imagens ocorreram durante um período de cinco dias ininterruptos, realizada no período que ocorreria o carnaval de 2021, compreendido do dia 13 ao dia 17 de fevereiro. As datas se referem ao fim de semana que antecede ao feriado de carnaval, a data comemorativa do carnaval e ao feriado da quarta-feira de cinzas. Era verificado se as datas das postagens estavam dentro do período de estudo. Nos casos afirmativos aplicavam-se os critérios de exclusão, que foram perfis de acadêmicos de outras Instituições de Ensino Superior (IES), docentes, clínicas e empresas de materiais odontológicos.

Para a sistematização da análise do material coletado, verificou-se a existência do comportamento individual ou coletivo que descumpriam o Decreto Estadual No 33.617/2020 que determinou o uso obrigatório de máscaras por todos aqueles que precisarem sair de suas residências; e o Decreto n. 33.927, que proibia eventos e atividades coletivas de qualquer natureza com aglomeração de pessoas durante o período de carnaval. O material coletado foi também categorizado quanto ao gênero do usuário da rede social, representação do usuário consumindo ou não bebidas alcóolicas, dia e horário em que a foto foi registrada, classificando em diurno, noturno e dia da semana.

Os dados foram coletados e registrados em planilhas do Microsoft Excel, os nomes dos acadêmicos foram substituídos por números, a fim de garantir o anonimato dos perfis analisados. Os dados foram expressos por meio de estatística descritiva quanto à frequência absoluta, média e porcentagem.

A Infodemiologia deve exercer comportamento ético sobre as informações de acesso aberto de indivíduos. Dessa forma, no presente estudo, adotou-se o método passivo, sem envolver ativamente os usuários, não necessitando 
aprovação do comitê de ética, já que todas as postagens no Instagram podem ser vistas por qualquer pessoa, a menos que o usuário altere o padrão da conta como privado.

Ressalta-se que a presente pesquisa respeita a Constituição da República Federativa do Brasil (1988) em Artigo 5, inciso X, e os artigos 20 e 21 do vigente Código Civil, pois não houve divulgação de imagens, não foi violada a intimidade, a vida privada, a honra, a imagem, a boa fama ou a respeitabilidade do sujeito. Bem como, consideramos que a análise de uma imagem publicada pelo usuário não configura e-crime.

Considerando os Direitos Autorais da difusão de imagens, a política de dados do Instagram estabelece que ao criar uma conta o usuário deve ponderar com quem escolhe compartilhar suas atividades, porque as postagens podem ser compartilhadas por terceiros dentro e fora da plataforma. O usuário de uma conta pública permite esses compartilhamentos (Instagram, 2021).

\section{RESULTADOS}

Dos 223 acadêmicos seguidores, foram identificados 47 perfis públicos (21\%), desses, 12 (26\%) apresentaram algum comportamento de risco (Figura 1). Sendo, 3 (25\%) pertenciam a contas masculinas e 9 (75\%) a femininas. Com base nesses perfis, foram contabilizadas 19 fotos, que caracterizavam o descumprimento dos decretos estaduais.

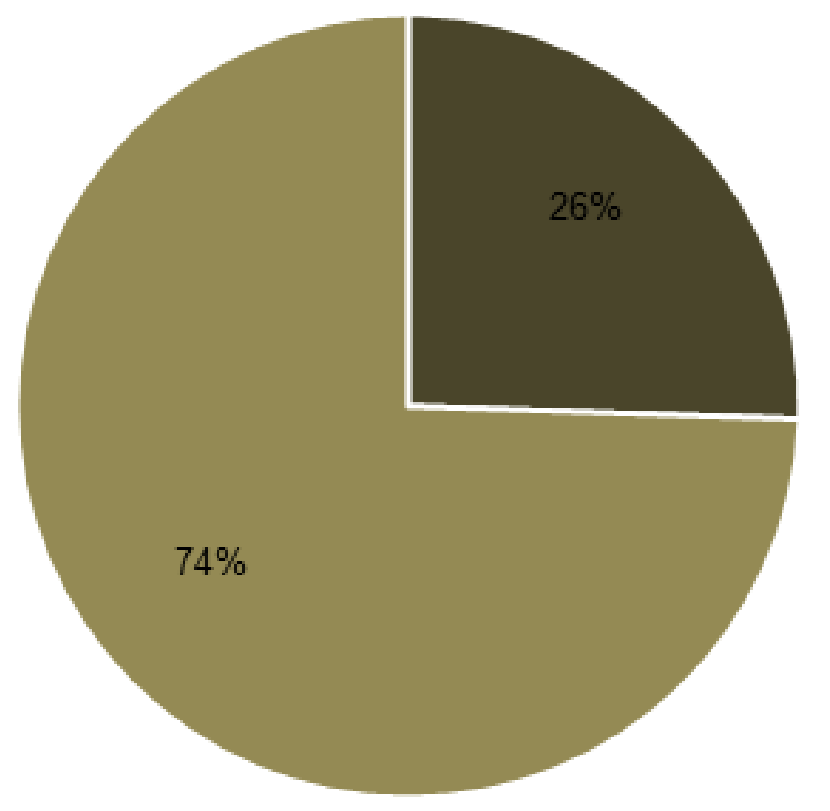

\section{- Perfis com comportamento de risco}

\section{- Perfis sem comportamento de risco}

Figura 1. Perfis de acadêmicos de odontologia e seus comportamentos em relação aos decretos de prevenção da covid-19 do Governo do Estado do Ceará.

Mesmo sendo a minoria da amostra estudada, os acadêmicos identificados adotando comportamento de risco podem ter sido potenciais disseminadores ou se contaminaram com SARS-CoV-2.

Um grande conjunto de dados não é um requisito obrigatório desde que os dados coletados representem o universo pesquisado. Devendo ser levado em consideração se a base de dados forneceu elementos potencialmente úteis para o apoio a tomada de decisão. Além do mais, grande volume de dados impossibilitaria uma análise fiel da realidade (Carvalho e Dallagassa, 2014; L. R. L. Silva e Botelho-Francisco, 2018).

Os principais meios de transmissão do SARS-CoV-2 são os fluidos, por isso o risco ocupacional de contágio é alto na Odontologia. Os docentes e discentes da área da saúde, durante os procedimentos que necessitam do contato próximo com os pacientes, estão mais susceptíveis às gotículas oronasais, tornando-se mais fácil a transmissibilidade e contágio da COVID-19. Com isso, esses profissionais podem se tornar potenciais portadores e disseminadores da doença. Isso remete à reflexão de que, ao retornarem aos campos de práticas e estágios, os docentes e discentes dos cursos da área da saúde também estarão expostos a esse mesmo risco (Maciel et al., 2020; Okyere et al., 2020; Oliveira et al., 2020).

Dentre os comportamentos de risco observados, o não uso de máscara e o distanciamento social foram as medidas mais descumpridas (Figura 2). 


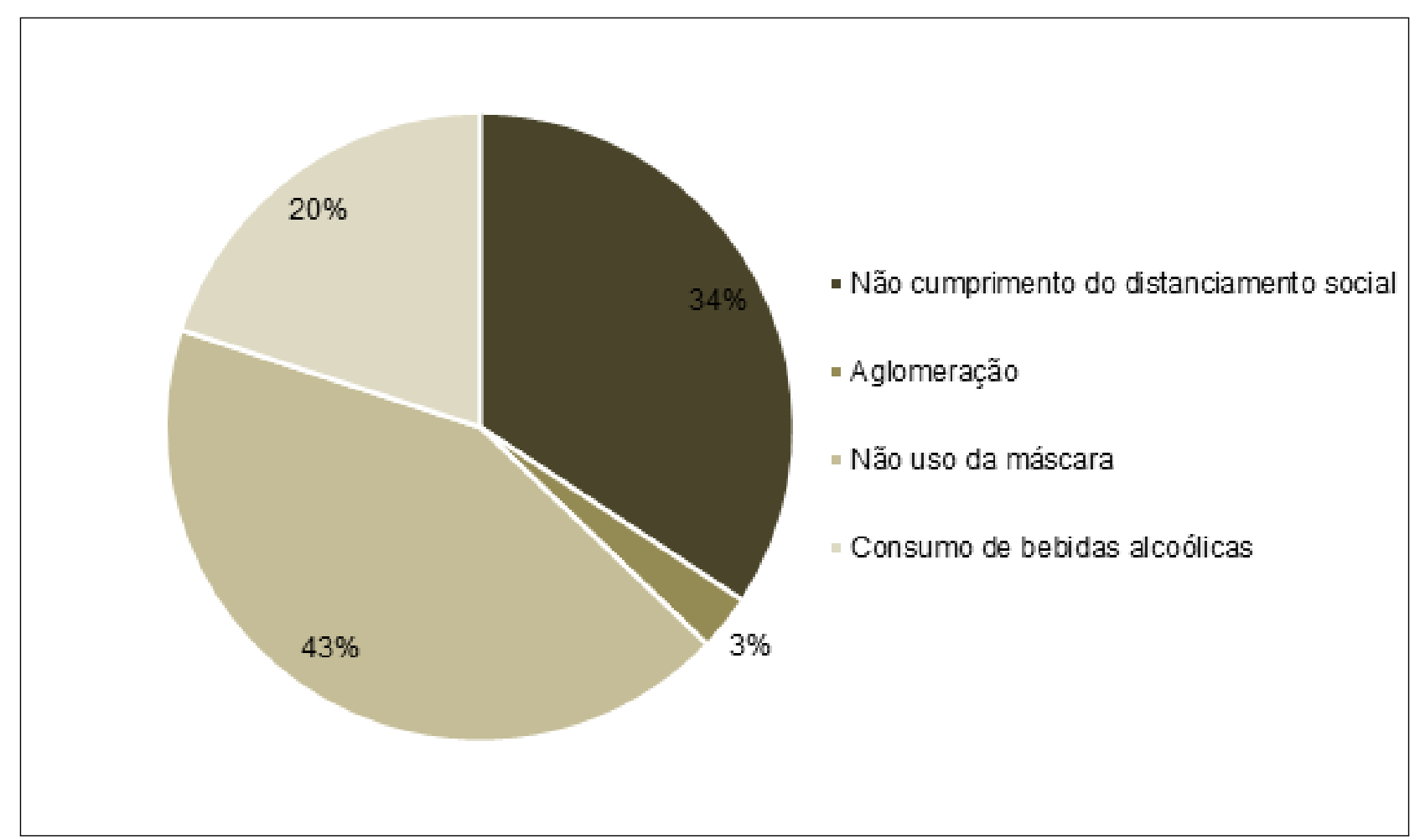

Figura 2. Comportamentos de riscos identificados em perfis de acadêmicos de Odontologia, em relação à disseminação e a resposta imunológica vinculados a COVID-19.

Os usuários do Instagram devem ter consciência de que suas postagens podem influenciar a atitude dos demais usuários e que toda a informação publicada em plataformas de Redes Sociais por um indivíduo em particular é representativa do modo de ser da pessoa em questão. As postagens representam o indivíduo e podem continuar representando no futuro. Portanto, o que é postado poderá refletir na sua imagem futuramente (Machado, 2016; ConnectSafely, 2021).

Já é senso comum que distanciamento social, isolamento e quarentena da população constituem medidas mais importantes e eficazes de controle do avanço do vírus. Faz-se necessário que as medidas preconizadas pelos órgãos oficiais sejam adotadas, mas, a implementação do distanciamento social na realidade brasileira é um grande desafio (Aquino et al., 2020; Ceará, 2020a; K. F. Santos e Barbosa, 2020).

Mesmo com a veiculação de campanhas sobre medidas preventivas, por algum motivo, a população brasileira apresenta resistência a adotar medidas simples para conter o avanço da disseminação da doença e preservar a saúde da população.

Araujo e Oliveira (2020) lançam luz sobre isso, sugerindo que seja decorrente a postura de negação, adotada por parte da população, às informações divulgadas por organizações mundiais consolidadas, instituições produtoras ou disseminadoras de conhecimento com base em evidências científicas, preferindo acreditar apenas neles mesmos ou em personalidades sem prestígio científico no campo da saúde que respondam de maneiras simples e imediata a fenômenos sociais complexos. Desde o início da pandemia no Brasil houve ampla veiculação de informações, tanto seguras quanto falsas, relacionadas à ciência, que podem ter influenciado no comportamento dos brasileiros no contexto da pandemia.

A divulgação de falas e pronunciamentos cujos teores estão em desacordo com estudos científicos muitas vezes é feita reforçando o caráter incorreto das informações, disseminando informações sem respaldo, mas por mais que sejam infundadas, têm o seu espaço. Quanto mais a população for exposta a informações falsas, mais distorcida será sua percepção da pandemia e mais relapso será o seu cumprimento das medidas de prevenção (P. V. D. Silva, 2020).

A população deve entender que medidas simples de prevenção estão relacionadas a fenômenos mais amplos, vinculadas à saúde pública, respaldadas por evidências científicas e organizações mundiais consolidadas, e isso deve estar acima de qualquer que seja o posicionamento político-ideológico. Neste cenário, novos hábitos embasados em evidências científicas devem ser adotados para controlar a disseminação, o contágio e salvar vidas (Araujo e Oliveira, 2020; França et al., 2020; F. C. C. Silva, 2020).

Sabe-se que medidas amplas de distanciamento social, principalmente aquelas que reduzem em pelo menos $60 \%$ os contatos sociais, têm o potencial de diminuir a transmissão da doença. Estas medidas devem ser fortalecidas e realizadas de forma intersetorial e coordenada entre as diferentes esferas governamentais e regiões para que seja alcançado o fim da epidemia o mais brevemente possível (Aquino et al., 2020). 
Após o período de estudo, o Governo do Estado do Ceará publicou o Decreto n. 33.936, o qual estabelece que até o dia 28 de fevereiro de 2021, permanecerão em vigor, no Estado do Ceará, as medidas de isolamento social previstas no Decreto n. 33.519, de 19 de março de 2020, e suas alterações posteriores. Em sequência, foi instituído o lockdown em todo o estado por meio do Decreto n. 33.965 (Decreto n. 33.936, 2021; Decreto n. 33.519, 2020; Decreto n. 33.965).

Esta medida pode estar associada à necessidade de um período de quarentena domiciliar de 14 dias que pode prevenir a propagação. Os acadêmicos que descumpriram as medidas preventivas preconizadas deveriam adotar a estratégia de quarentena domiciliar a fim de prevenir a propagação viral.

Entretanto, o convívio domiciliar com pacientes positivos para COVID-19 é um fator de risco. A contaminação pode ocorrer em qualquer momento ao longo dos períodos de 14 dias, os assintomáticos podem apresentar sintomas após sete dias de contato domiciliar. No caso de pessoas que não possam cumprir a quarentena de 14 dias, devem continuar a evitar contato próximo com outras pessoas e adotar o hábito de usar máscaras até 14 dias após o início dos sintomas, pois o período de incubação do novo coronavírus pode durar até 14 dias, além de que o vírus pode permanecer por até 24 dias na saliva. Em vista disso, um período de quarentena mais curto pode permitir a transmissão contínua da doença (Riatto, de Queiroz Vanderlei, Cabral, e Galvão, 2020; Rolfes, 2021).

A maioria das pessoas que apresentam resultados de teste positivos para a COVID-19 são assintomáticos. Os pacientes mais velhos com mais comorbidades apresentam maiores chances de desenvolver doença na forma sintomática, em sua maioria evoluem para a intubação e morrem. Além disso, esses pacientes permaneceram por mais tempo na Unidade de Terapia Intensiva (UTI) e intubação mais prolongada em comparação com os pacientes sem comorbidades (Atherstone et al., 2021; Maltezou et al., 2021).

A pandemia da COVID-19 gera momentos de medos e inseguranças. O consumo de bebidas alcoólicas pode estar associado à experiência de episódios traumáticos relacionados à COVID-19. É possível esperar um aumento dos padrões de consumo de álcool posteriores à pandemia, trazendo mortalidade e morbidade associadas (Garcia e Sanchez, 2020; Oliveira et al., 2020).

Foram identificados $3 \%$ de perfis de acadêmicos que adotavam o comportamento de consumo de bebidas alcoólicas, ocorrendo principalmente entre os perfis do gênero feminino, sendo que o mesmo perfil, em alguns casos, apresentou recorrência desse comportamento de risco. Com a proibição do funcionamento de bares, restaurantes, casas noturnas e outros estabelecimentos, o consumo de bebidas alcoólicas, que era realizado em espaços públicos, passou ao privado, tendo o domicílio se tornado o local de escolha para esse comportamento, podendo contribuir no aumento desse comportamento de risco. Vem sendo evidenciado internacionalmente aumento no consumo de bebidas alcoólicas em casa, aparentemente decorrente do isolamento, e na contramão das evidências sobre os danos associados (Barbosa, Gomes, Gomes, e Souza, 2020; Garcia e Sanchez, 2020; Jesus et al., 2017; Soares et al., 2020).

Sabe-se que o consumo de bebidas alcoólicas é um hábito cultural brasileiro e que o isolamento social, ao mesmo tempo que pode colaborar para a redução do consumo, pode também fomentar situações capazes de aumentá-lo (Barbosa et al., 2020; Jesus et al., 2017).

As mídias possuem como objetivo influenciar as pessoas a adotarem um determinado comportamento. Estas podem ser um veículo de ações direcionadas à saúde pública na área de prevenção ao consumo abusivo de álcool durante a pandemia, mas o que se observou foi que, durante o período de distanciamento social, a difusão midiática de lives de artistas e celebridades em plataformas de Redes Sociais estimularam o consumo de bebidas alcoólicas (Chagas, Paula, e Martins, 2020; Soares et al., 2020).

O consumo de bebidas alcoólicas impacta de forma diferente em cada pessoa, sabe-se que, de uma maneira geral, este hábito pode ocasionar diversas complicações imunológicas, tornando os consumidores mais propícios a infecções, devido à inibição dos quimiotáxicos atrativos de leucócitos e perda parcial da capacidade das respostas imunitárias, aumento de infecções e aumentando a predisposição a diabetes, hipertensão, gastrite e osteoporose. Por fragilizar o sistema imune, além de ocasionar agravos diretos a vários órgãos, o consumo de bebidas alcoólicas pode aumentar as chances do desenvolvimento dos sintomas mais graves da COVID-19 (Barbosa et al., 2020; Garcia e Sanchez, 2020; Jesus et al., 2017).

As postagens em redes sociais são uma forma de influenciar o comportamento dos demais usuários (Instagram, 2021). Ramos e Cunha (2018) relataram que os estudantes universitários da área da saúde são influenciados por outros estudantes a consumirem bebidas alcoólicas. O consumo se dá em busca da sensação de prazer advinda dos efeitos do álcool no organismo ou para ajudar nas interações sociais.

Isolamento social e COVID-19 são temas recentes, por isso a realização de mais estudos sobre os temas são necessárias, para que haja uma melhor compreensão das alterações biopsicossociais na população brasileira. Além disso, medidas de conscientização e educação para consumo de álcool a fim de evitar a progressão no padrão de beber como consequência dos efeitos da pandemia (Garcia e Sanchez, 2020; Ramos e Cunha, 2018). 
Apesar do momento trazer sentimentos de medos e inseguranças, são necessárias mudanças de comportamento para a contenção do coronavírus. O profissional de saúde em formação, deve conciliar sua vida pessoal com toda a sobrecarga sobre sua futura atuação profissional. Devendo repensar as relações sociais, biológicas, psicológicas e adotar comportamentos de enfrentamento à pandemia que está longe de ter seu fim (Oliveira et al., 2020).

Todos os envolvidos com serviços odontológicos terão que se adaptar a um método mais rigoroso de proteção da equipe e dos pacientes, para garantir um atendimento seguro e resolutivo, e que terá impactos futuros nas novas formações e atualizações da equipe de saúde bucal (Maciel et al., 2020).

Os comportamentos de risco variaram ao longo dos dias analisados, sendo que o domingo e a quarta-feira de cinzas apresentaram maior número. Chama atenção a inexistência de comportamentos de risco na segunda-feira (Figura 3). Em relação ao período, a maioria das fotos foram registradas no período diurno (8), apenas 1 no período noturno, as demais não permitiram a identificação do período.

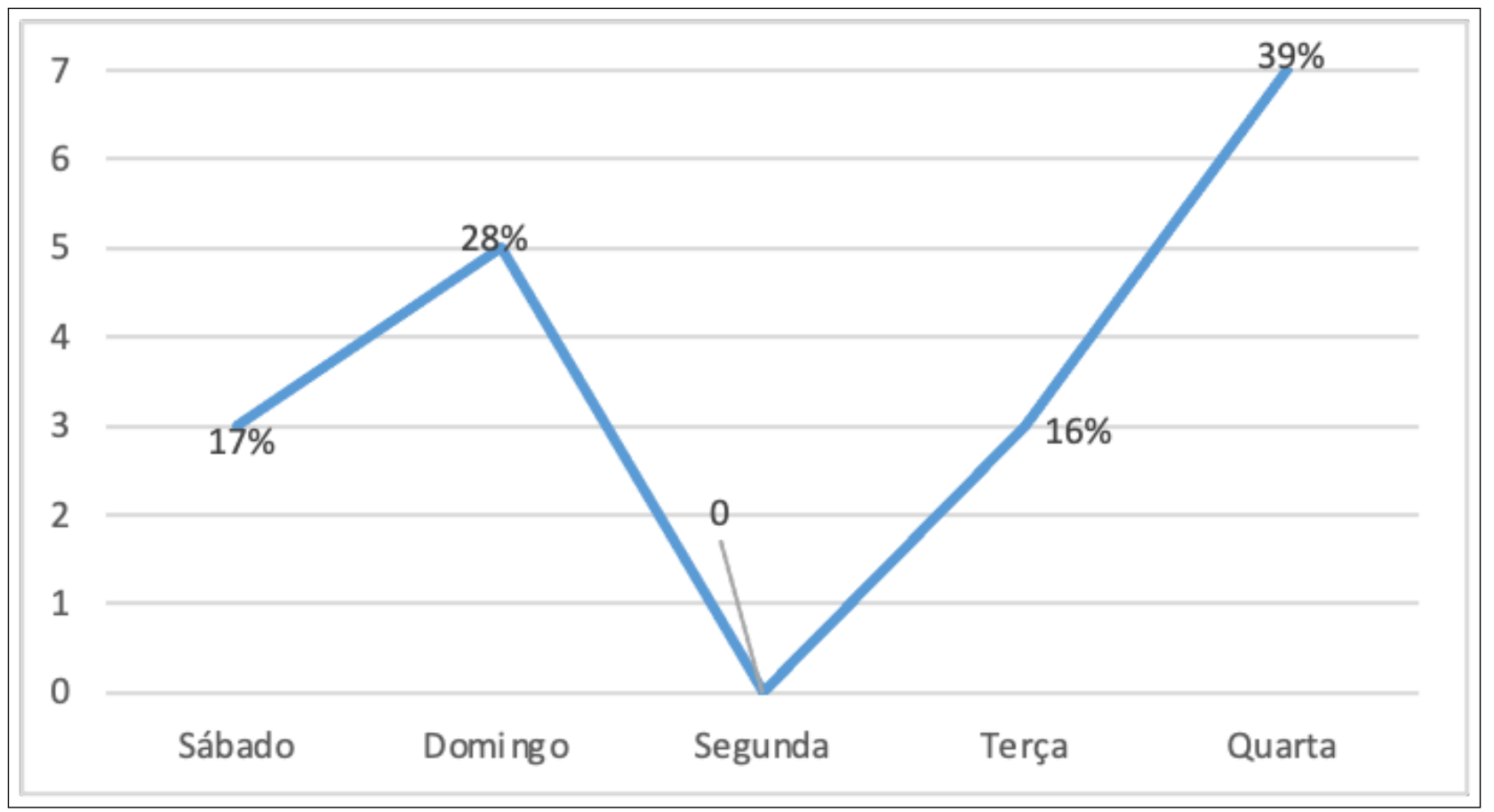

Figura 3. Dias com presença de comportamentos de riscos nos perfis de acadêmicos de odontologia em relação aos decretos de prevenção da covid-19 do Governo do Estado do Ceará.

Observou-se que o comportamento de risco ocorreu em $80 \%$ dos dias analisados, sempre sendo identificados os três comportamentos: aglomerações, consumo de álcool e o não uso da máscara. No sábado houve média de 2 dos comportamentos de risco por perfil, no domingo 1,8, na terça 1,4 e na quarta 2,4 .

A postura pessoal e profissional, os hábitos e as rotinas dentro e fora do consultório odontológico devem ser repensadas, a fim de buscar a segurança tanto no período da pandemia da COVID-19 quanto no período pós-pandemia. Por este motivo, acadêmicos de Odontologia devem adotar medidas de prevenção para não serem contaminados ou contaminar seus pacientes.

Devemos levar em consideração que os comportamentos evidenciados em plataforma de Redes Sociais indicam posturas e condutas de um indivíduo, mas não podem ser determinantes de que essas condutas serão reproduzidas em ambiente acadêmico e profissional. É preciso separar os comportamentos para não realizar um julgamento moral de condutas individuais não relacionadas ao exercício profissional. No entanto, não diminuindo a responsabilidade individual de adoção de comportamentos preventivos de disseminação potencial de agentes infecciosos.

Os docentes e discentes da área da saúde durante os procedimentos que necessitam o contato próximo com os pacientes, clientes ou usuários estão mais susceptíveis às gotículas oro nasais, provenientes da saliva, espirro, tosse, tornando-se mais fácil a transmissibilidade e contágio da COVID-19. Nessa circunstância, uma rotina rigorosa de biossegurança deve sempre ser adotada caracterizando todo paciente com um potencial agente propagador de infecções cruzadas. Isso remete à reflexão de que, ao retornarem aos campos de prática e estágios, os docentes e discentes dos cursos da área da saúde também estarão expostos a esse mesmo risco (Maciel et al., 2020; Okyere et al., 2020; Oliveira et al., 2020).

A prática clínica odontológica, principalmente nas clínicas-escola das faculdades e universidades, que dispõem de vários equipamentos em funcionamento simultâneo, gera gotículas e aerossóis na maioria dos procedimentos, 
os quais se misturam com fluidos como a saliva e o sangue, lançando no ar e ficando suspensos por longos períodos até aderirem a alguma superfície, onde podem permanecer por 9 dias, ou podendo ser inalados por indivíduos sadios. Portanto, a biossegurança mediante controle severo de limpeza, desinfecção e correto uso dos Equipamentos de Proteção Individual (EPI) devem ser seguidos rigorosamente (Riatto et al., 2020). Critérios mais rigorosos de biossegurança foram implementados na Odontologia com o surgimento da COVID-19, mas ainda não existem evidências bem estabelecidas, cabe ao profissional adotar todas as medidas preventivas à disposição para minimizar as contaminações (Faria et al., 2020).

O exercício profissional docente traz consigo diversas responsabilidades, dentre elas o compromisso de formar um cidadão capaz de atuar no meio em que vive e contribuir para a solução de problemas sociais, quaisquer que sejam as suas dimensões. Os exemplos dados pelos profissionais mais experientes permitem aos acadêmicos desenvolver diversos aprendizados e práticas. Estas oportunidades de crescimento, amadurecimento e desenvolvimento profissional, pessoal e acadêmico podem ser repensadas e reforçadas durante o período de pandemia causada pela COVID-19. Medidas de proteção aos indivíduos são necessárias tanto no período da pandemia da COVID-19, quanto no período pós-pandemia (Maciel et al., 2020; Marta et al., 2020; Oliveira et al., 2020).

A Odontologia é uma das áreas de maior risco de infecção. Nessa circunstância, destaca-se o papel do docente no que se refere à fiscalização e atualização frequentes de práticas e conhecimento das medidas preventivas e de proteção adequadas que permitam o exercício de atividades acadêmicas em segurança. Diante disso, os cirurgiões-dentistas podem contribuir com suas expertises, neste momento crítico, com a adoção de medidas preventivas (K. F. Santos \& Barbosa, 2020).

Espera-se que os acadêmicos que adotaram comportamentos de risco, ao menos adotem comportamentos preventivos durante o atendimento à comunidade, tais como os indicados por Oliveira et al. (2020) como reforçar a limpeza de superfícies de instrumentais, bancadas, armários, torneiras, cadeiras, maçanetas de portas; realizem, frequentemente, a higiene das mãos com água e sabão líquido ou preparação alcoólica a 70\% em dispensador; utilizem papel toalha e o descartem em lixeira com pedal; além da disponibilização de suportes com preparação de álcool em gel a 70\% nas salas de espera, entradas das salas de aula, laboratórios, consultórios e clínicas.

Na prática é preciso que muitas medidas adicionais sejam implementadas, como testes prolongados para infecções, a ampliação da sua capacidade para identificar indivíduos infectados com formas sintomáticas, pré-sintomáticas e assintomáticas, definição precisa dos casos suspeitos e confirmados, o mascaramento universal e higiene das mãos em todos os momentos para reduzir o risco de transmissão viral. Tais normas devem continuar até que a COVID-19 seja totalmente erradicada (Aquino et al., 2020; Atherstone et al., 2021; Meidan et al., 2021).

Os acadêmicos de Odontologia dos períodos mais avançados apresentaram melhores condutas em relação a agentes infecciosos, mas há negligência das medidas de proteção (Freitas, Carvalho, Alves, da Silva Costa, \& Carvalho, 2021). Assim, espera-se que os acadêmicos assumam posturas preventivas em relação à pandemia.

A infodemiologia para planejamento e ação em saúde é promissora, pois já demonstrou ser útil no rastreamento e previsão de surtos, epidemias e pandemias na Europa, inclusive na COVID-19, podendo ser uma ferramenta para o controle no Brasil, entretanto, devem ser utilizados com cautela e de forma complementar aos estudos populacionais convencionais (Aquino et al., 2020; Mavragani et al., 2018; Soreni, Cameron, Streiner, Rowa, e McCabe, 2019).

Encerramos com as palavras de Lehmkuhl e da Silva (2020) quando dizem que números podem contar fatos e que chegar o mais próximo do contexto real é objetivo da ciência em prol da vida, da qualidade de vida e todo o seu desenvolvimento.

\section{CONCLUSÃO}

Foram obtidos dados valiosos do comportamento social em tempo real durante a pandemia do COVID-19 no Estado do Ceará, por meio da análise de imagens publicadas na rede social Instagram sobre o comportamento social de acadêmicos de Odontologia durante o período de feriado de carnaval.

Apesar das imagens postadas evidenciarem comportamentos de riscos em um contexto pandêmico, os dados apresentados são animadores devido ao reduzido número de acadêmicos de Odontologia adotando comportamento de risco. As postagens simbolizam ações de indivíduos em um determinado momento, mesmo trazendo dados valiosos à realidade, e pode sofrer mudanças ao longo do tempo, permitindo análises posteriores para verificar se há um padrão de comportamento.

Embora os dados apresentados sejam relevantes, os perfis privados foram uma limitação para o estudo, não permitindo analisar o comportamento de todos os acadêmicos que se encontram em disciplinas de atendimento odontológico prático. O acesso a esses perfis poderia apresentar outra realidade.

Vale ressaltar que, mesmo com o comprometimento das instituições governamentais e de ensino em adotar medidas preventivas de biossegurança, cabe aos acadêmicos da área da saúde adotar medidas preventivas em 
relação à infecção e à transmissão da COVID-19 a seus familiares e pacientes. Essas medidas devem ser adotadas pela população em geral, por acadêmicos e profissionais de saúde para contribuir com a redução da disseminação do vírus, esses comportamentos serão necessários mesmo após a implantação de programas de vacinação para a população. O comportamento individual contribui para que o fim da epidemia ocorra o mais brevemente possível.

Apesar dos dados não poderem ser generalizados, consideramos que a metodologia adotada pode ser usada como uma ferramenta de gestão para associar e prever agravos em saúde. Esperamos com este breve estudo contribuir para o melhor entendimento sobre a busca por informações de saúde por meio do comportamento em tempo real a partir de postagens em plataformas de redes sociais. Como também, promover a conscientização dos acadêmicos da área da saúde para a adoção de medidas preventivas direcionadas para evitar a disseminação da COVID-19.

Os resultados mostram-se relevantes por analisar acontecimentos locais e as peculiaridades regionais dos comportamentos de acadêmicos da área da saúde e podem ser utilizados como elementos de apoio à decisão. Os indicadores apresentados são relativos e refletem o comportamento de uma amostra de acadêmicos de Odontologia de um centro universitário em particular, não podendo ser generalizados. Mais informações ainda são necessárias para uma análise profunda, sugerimos que a metodologia seja reproduzida em contextos semelhantes, podendo ser acrescentado análise de mensagens, compartilhamentos e hashtags. 


\section{REFERÊNCIAS}

Aquino, E. M., Silveira, I. H., Pescarini, J. M., Aquino, R., Souza-Filho, J. A. d., Rocha, A. d. S., .. others (2020). Medidas de distanciamento social no controle da pandemia de covid-19: potenciais impactos e desafios no brasil. Ciência ES Saúde Coletiva, 25, 2423-2446. doi: 10.1590/141381232020256.1.10502020

Araujo, R. F., \& Oliveira, T. M. (2020). Desinformação e mensagens sobre a hidroxicloroquina no twitter: da pressão política à disputa científica. AtoZ: novas práticas em informação e conhecimento, 9(2), 196-205. doi: 10.5380/atoz.v9i2.75929

Atherstone, C., Peterson, M. L., Malone, M., Honein, M. A., MacNeil, A., O'Neal, C. S., ... others (2021). Time from start of quarantine to sars-cov-2 positive test among quarantined college and university athletes. Morbidity and Mortality Weekly Report, 70(1), 7. Recuperado de https://www.cdc.gov/mmwr/volumes/70/wr/mm7001a2 .htm\# contribAff

Barbosa, D. J., Gomes, M. P., Gomes, A. M. T., \& Souza, F. B. A. (2020). Relação entre o consumo de drogas psicoativas e covid-19. Journal of Management $\& 3$ Primary Health Care, 12, 1-9. doi: 0.14295/jmphc.v12.1000

Bragazzi, N. L., Bacigaluppi, S., Robba, C., Siri, A., Canepa, G., \& Brigo, F. (2016). Infodemiological data of west-nile virus disease in italy in the study period 2004-2015. Data Brief , 9, 839-845. doi: 10.1016/j.dib.2016.10.022

Carvalho, D. R., \& Dallagassa, M. R. (2014). Mineração de dados: aplicações, ferramentas, tipos de aprendizado e outros subtemas. AtoZ: novas práticas em informação e conhecimento, 3(2), 82-86. Recuperado de http://www.atoz .ufpr.br/index.php/atoz/article/view/93

Chagas, C., Paula, T. C. S., \& Martins, L. B. (2020). O aumento do consumo de álcool em tempos de pandemia: mídia e normas sociais: Artigo de opinião. Comunicação em Ciências da Saúde, 31, 116-120. Recuperado de http://www.escs.edu.br/revistaccs/index.php/ comunicacaoemcienciasdasaude/article/view/718/397

ConnectSafely. (2021). Instagram. Recuperado de https:// about.instagram.com/community/safety

Constituição da República Federativa do Brasil. (1988). Constituição da república federativa do brasil de 1988. Recuperado de http://www.planalto.gov.br/ccivil_03/ constituicao/constituicao.htm

Decreto n. 33.519. (2020). Intensifica as medidas para enfrentamento da infecção humana pelo novo coronavírus no estado do ceará. Ceará. Recuperado de https://www.cge .ce.gov.br/decretos-estaduais/

Decreto n. 33.927. (2021). Estabelece, no estado do ceará, novas medidas preventivas à disseminação da covid-19, no período de carnaval, e dá outras providências. Ceará. Recuperado de https://www.cge.ce.gov.br/decretos-estaduais/

Decreto n. 33.936. (2021). Prorroga o isolamento social e estabelece medidas preventivas direcionadas a evitar a disseminação da covid-19, no estado do ceará, e dá outras providências. Ceará. Recuperado de https://www.cge.ce .gov.br/decretos-estaduais/

Decreto n. 33.965. (2021). Restabelece, no estado do ceará, a política de isolamento social rígido como medida de enfrentamento à covid - 19, e dá outras providências. Ceará. Recuperado de https://www.cge.ce.gov.br/decretos-estaduais/

Eysenbach, G. (2009). Infodemiology and infoveillance: framework for an emerging set of public health informatics methods to analyze search, communication and publication behavior on the internet. Journal of medical Internet research, 11(1), 1-10. doi: 10.2196/jmir.1157

Faria, M. H. D., Pereira, L. D., Limeira, A. B. P., Dantas, A. B. S., de Oliveira Moura, J. M. B., \& de Almeida, G. C. M. (2020). biossegurança em odontologia e covid-19: Uma revisão integrativa. Cadernos ESP-Revista Científica da Escola de Saúde Pública do Ceará, 14(1), 53-60. Recuperado de https://cadernos.esp.ce.gov.br/index.php/cadernos/ article/view/335

França, F. P., Oliveira Araújo, D., \& Silva, M. B. (2020). A iniciativa digital convide-i9 no combate à infodemia de covid-19: breves apontamentos de atuação. AtoZ: novas práticas em informação e conhecimento, 9(2), 248-252. doi: 10.5380/atoz.v9i2.76472

Freitas, J. A., Carvalho, I. S., Alves, F. B., da Silva Costa, N., \& Carvalho, G. A. O. (2021). Biossegurança em procedimentos cirúrgicos odontológicos frente a pandemia do covid-19. Research, Society and Development, 10(1). doi: 10.33448/rsd-v10i1.11401

Garcia, L. P., \& Sanchez, Z. M. (2020). Consumo de álcool durante a pandemia da covid-19: uma reflexão necessária para o enfrentamento da situação. Cadernos de Saúde Pública, 36, 1678-4464. doi: 10.1590/0102-311x00124520

Grimaldi, S. S. L., Rosa, M. N. B., Loureiro, J. M. M., \& Oliveira, B. F. d. (2019). O patrimônio digital e as memórias líquidas no espetáculo do instagram. Perspectivas em Ciência da Informação, 24, 51-77. Recuperado de https://www.scielo.br/j/pci/a/ q5jjt6BT3CZbmpxLZmrLNrK/?lang=pt\&format=pdf

Inomata, D. O., dos Passos, K. G. F., Pintro, S., Sena, P. M. B., \& Bedin, J. (2020). Compartilhamento de conhecimentos e aprendizagem colaborativa em tempo de pandemia. AtoZ: novas práticas em informação e conhecimento, 9(2), 206-215. doi: 10.5380/atoz.v9i2.76168

Instagram. (2021). Política de dados do instagram. Recuperado de https://www.facebook.com/help/instagram/ 519522125107875

Jesus, J. R., Santos, S., Menezes, A. N., Santos, C. A., Silva, K. B., \& Silva, S. A. A. (2017). Efeitos deletérios do álcool no sistema imunológico. Universidade Tiradentes. Recuperado de https://eventos.set.edu.br/sempesq/article/ viewFile/7672/3496

Lehmkuhl, C. S., \& da Silva, E. C. L. (2020). Os registros civis em meio à pandemia de covid-19 no brasil: Releituras. AtoZ: novas práticas em informação e conhecimento, 9(2), 129-138. doi: 10.5380/atoz.v9i2.76908

Machado, L. M. O. (2016). A influência da informação publicada nas "redes sociais" no processo de seleção e recrutamento: um estudo exploratório na literatura. AtoZ: novas práticas em informação e conhecimento, 5(1), 10-20. doi: 10.5380/atoz.v5i1.44035

Maciel, Y. L., da Silva Sobrinho, A. R., \& Medrado, J. d. G. B. (2020). Covid-19 na prática odontológica: A renovação da biossegurança no consultório. Scientific-Clinical Odontology, 19(3), 243-247. Recuperado de https://cro-pe.org.br/site/adm_syscomm/publicacao/ foto $/ 158$.pdf $\#$ page $=37$

Maltezou, H. C., Raftopoulos, V., Vorou, R., Papadima, K., Mellou, K., Spanakis, N., ... others (2021). Association between upper respiratory tract viral load, comorbidities, disease severity, and outcome of patients with sars-cov-2 
infection. The Journal of infectious diseases, 223(7), 11321138. doi: 0.1093/infdis/jiaa804

Marta, C. B., da Silva, W. B. H., Côrtes, E. M. P., Machado, T. O., Francisco, M. T. R., da Silva, P. O., ... das Neves, M. P. (2020). Telemonitoramento: análise da percepção dos acadêmicos de enfermagem frente à pandemia da covid19. Global Academic Nursing Journal, 1(3), e52-e52. doi: $10.5935 / 2675-5602.20200052$

Mavragani, A., Ochoa, G., \& Tsagarakis, K. P. (2018). Assessing the methods, tools, and statistical approaches in google trends research: systematic review. Journal of Medical Internet Research, 20(11), e270. doi: 10.2196 jmir.9366

Meidan, D., Schulmann, N., Cohen, R., Haber, S., Yaniv, E., Sarid, R., \& Barzel, B. (2021). Alternating quarantine for sustainable epidemic mitigation. Nature communications, 12(1), 1-12. doi: 10.1038/s41467-020-20324-8

Okyere, I., Chuku, E. O., Ekumah, B., Angnuureng, D. B., Boakye-Appiah, J. K., Mills, D. J., ... Crawford, B. (2020). Physical distancing and risk of covid-19 in small-scale fisheries: a remote sensing assessment in coastal ghana. Scientific reports, 10(1), 22407. doi: 10.1038/s41598-020-79898-4

Oliveira, Z. M., de Freitas, L. M. A., dos Santos, N. C. N., Dias, J. A. A., de Freitas, M. d. C. A., \& Oliveira, T. M. (2020). Estratégias para retomada do ensino superior em saúde frente a covid-19. Revista Enfermagem Atual In Derme, 93, e020008-e020008. Recuperado de https://www.revistaenfermagematual.com/index.php/ revista/article/view/803/678

Organização Mundial de Saúde. (2021). Painel do coronavírus da oms (covid-19). Recuperado de https:// covid19.who.int/

Pires, G. T. S. (2013). Fotografia através de dispositivos móveis: Estudo de caso sobre o instagram. Revista da Graduação, 6(1). Recuperado de https://revistaseletronicas .pucrs.br/index.php/graduacao/article/view/13776

Ramos, L. C. S., \& Cunha, R. A. (2018). Perfil do consumo de álcool entre os estudantes de medicina do campus lagarto da universidade federal de sergipe (Monografia de Graduação em Medicina, Universidade Federal de Sergipe, Sergipe, Brasil). Recuperado de https://ri.ufs.br/handle/riufs/9294

Rezer, R. M., Knoll, G. F., \& Ghisleni, T. S. (2018). Posts publicitários no instagram: análise de formatos, funções e conteúdo. Disciplinarum Scientia/ Artes, Letras e Comunicação, 19(1), 35-43. Recuperado de https://periodicos.ufn .edu.br/index.php/disciplinarumALC/article/view/2468

Riatto, S. G., de Queiroz Vanderlei, A. C., Cabral, G. M. P., \& Galvão, A. K. C. (2020). biossegurança no atendimento odontológico em clínica-escola em tempos de pós-pandemia por covid-19. Diálogos em Saúde, 3(1). Recuperado de https://periodicos.iesp.edu.br/ index.php/dialogosemsaude/article/view/276\#: : :text= A\%20doen\%C3\%A7a\%20covid\%2D19\%20causada,feitos\% 20simultaneamente $\% 20 \mathrm{em} \% 20$ ambiente $\% 20 \%$ C3\%BAnico

Rodrigues, J. V. (2018). A pesquisa etnográfica e a gramática dos sentimentos: Introdução à antropologia das emoções através das mídias sociais. bpgd. Recuperado de https://www.researchgate.net/profile/ Tarcizio-Silva-3/publication/327335155_Estudando

Cultura_e_Comunicacao_com_Midias_Sociais/

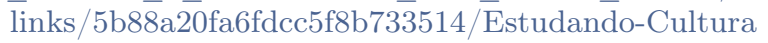
-e-Comunicacao-com-Midias-Sociais.pdf $\#$ page $=80$

Rolfes, M. A. (2021). Implications of shortened qua- rantine among household contacts of index patients with confirmed sars-cov-2 infection-tennessee and wisconsin, april-september 2020. MMWR. Morbidity and Mortality Weekly Report, 69(5152), 1633-1637. doi: 10.15585 / mmwr.mm695152a1

Santos, A. P., \& Nascimento, V. G. (2021). Ação cultural com mídias sociais: análise do facebook do sistema estadual de bibliotecas públicas de são paulo (siseb). AtoZ: novas práticas em informação e conhecimento, 10(1), 25-38. doi: 10.5380/atoz.v10i1.76615

Santos, K. F., \& Barbosa, M. (2020). Covid-19 e a odontologia na prática atual. Revista Eletrônica Acervo Saúde, 12(11). doi: 10.25248/reas.e5113.2020

Secretaria de Saúde do Estado do Ceará. (2021). Boletim epidemiológico novo coronavírus. Recuperado de https://indicadores.integrasus.saude.ce.gov.br/ indicadores/indicadores-coronavirus/coronavirus-ceara

Silva, F. C. C. (2020). Podcasts e webnars sobre covid19 na área de ciência da informação. AtoZ: novas práticas em informação e conhecimento, 9(2), 139-147. doi: 10.5380 /atoz.v9i2.75860

Silva, I. O., \& Gouveia, F. C. (2021). Engajamento informacional nas redes sociais: como calcular? AtoZ: novas práticas em informação e conhecimento, 10(1), 94-102. doi: 10.5380/atoz.v10i1.76633

Silva, L. R. L., \& Botelho-Francisco, R. E. (2018). Da representação ao monitoramento: a criação de uma ontologia do discurso de ódio online brasileiro. AtoZ: novas práticas em informação e conhecimento, 7(2), 28-33. doi: 0.5380 /atoz.v7i2.67243

Silva, P. V. D. (2020). Pandemia e infodemia nas mídias: análise da desordem informacional no twitter. AtoZ: novas práticas em informação e conhecimento, 9(2), 148-159. doi: 10.5380/atoz.v9i2.76506

Soares, J., Reinaldo, A. M. S., Gomes, N. M. R., Silveira, B. V., Pillon, S. C., \& Pereira, M. O. (2020). O consumo de substâncias psicoativas na pandemia de covid-19. Enfermagem em saúde mental e COVID-19, 34-44. doi: 10.51234/aben.20.e04.c05

Soreni, N., Cameron, D. H., Streiner, D. L., Rowa, K., \& McCabe, R. E. (2019). Seasonality patterns of internet searches on mental health: Exploratory infodemiology study. JMIR mental health, 6(4), e12974. doi: 10.2196/ 12974

Tana, J. (2019). Infodemiology: Studying rhythmicity in online health information behaviour. Abo Akademi University. Recuperado de https://www.doria.fi/handle/10024/173030

Xu, C., Wang, Y., Yang, H., Hou, J., Sun, L., Zhang, X., .. others (2019). Association between cancer incidence and mortality in web-based data in china: Infodemiology study. Journal of medical Internet research, 21(1), e10677. doi: $10.2196 / 10677$

Como citar este artigo (APA):

Monte, F. M. \& Girão, M. V. D. (2022). Análise do comportamento de acadêmicos de Odontologia em tempos de pandemia do novo coronavírus a partir das imagens do Instagram. AtoZ: novas práticas em informação e conhecimento, 11, 1 - 12. Recuperado de: http://dx.doi.org/10.5380/atoz.v11.80245 


\section{NOTAS DA OBRA E CONFORMIDADE COM A CIÊNCIA ABERTA}

\section{CONTRIBUIÇÃO DE AUTORIA}

\begin{tabular}{|l|c|c|}
\hline $\begin{array}{l}\text { Papéis } \\
\text { e contribuições }\end{array}$ & $\begin{array}{c}\text { Francisca Mariane } \\
\text { Martins Monte }\end{array}$ & $\begin{array}{c}\text { Mauro Vinícius } \\
\text { Dutra Girão }\end{array}$ \\
\hline Concepção do manuscrito & $\mathrm{X}$ & $\mathrm{X}$ \\
\hline Escrita do manuscrito & $\mathrm{X}$ & $\mathrm{X}$ \\
\hline Metodologia & $\mathrm{X}$ & $\mathrm{X}$ \\
\hline Curadoria dos dados & & $\mathrm{X}$ \\
\hline Discussão dos resultados & $\mathrm{X}$ & \\
\hline Análise dos dados & & \\
\hline
\end{tabular}

\section{Disponibilidade de Dados Científicos da Pesquisa}

Os conteúdos subjacentes ao texto da pesquisa estão contidos no manuscrito.

\section{EQUIPE EDITORIAL}

\section{Editora/Editor Chefe}

Maria do Carmo Duarte Freitas (https://orcid.org/0000-0002-7046-6020)

\section{Editora/Editor Associada/Associado}

Paula Carina de Araújo (https://orcid.org/0000-0003-4608-752X)

Helza Ricarte Lanz (https://orcid.org/0000-0002-6739-2868)

\section{Editora/Editor de Texto Responsável}

Byanca Neumann Salerno (https://orcid.org/0000-0002-1609-4684)

Cristiane Sinimbu Sanchez (https://orcid.org/0000-0002-0247-3579)

\section{Editora/Editor de Layout}

Felipe Lopes Roberto (https://orcid.org/0000-0001-5640-1573) 